\title{
La Mort du roi Arthur. Publication, traduction, présentation et notes par Emmanuèle Baumgartner et Marie-Thérèse de Medeiros
}

\section{Maria Colombo Timelli}

\section{(2) OpenEdition}

\section{Journals}

Édition électronique

URL : http://journals.openedition.org/studifrancesi/9109

DOI : 10.4000/studifrancesi.9109

ISSN : 2421-5856

Éditeur

Rosenberg \& Sellier

\section{Édition imprimée}

Date de publication : 1 juin 2008

Pagination : 158-159

ISSN : 0039-2944

\section{Référence électronique}

Maria Colombo Timelli, «La Mort du roi Arthur. Publication, traduction, présentation et notes par Emmanuèle Baumgartner et Marie-Thérèse de Medeiros », Studi Francesi [En ligne], 154 (LII | I) | 2008, mis en ligne le 30 novembre 2015, consulté le 08 janvier 2021. URL : http://journals.openedition.org/ studifrancesi/9109; DOI : https://doi.org/10.4000/studifrancesi.9109

Ce document a été généré automatiquement le 8 janvier 2021.

\section{cc) (†) $\odot$}

Studi Francesi è distribuita con Licenza Creative Commons Attribuzione - Non commerciale - Non opere derivate 4.0 Internazionale. 


\title{
La Mort du roi Arthur. Publication, traduction, présentation et notes par Emmanuèle Baumgartner et Marie-Thérèse de Medeiros
}

\author{
Maria Colombo Timelli
}

\section{RÉFÉRENCE}

La Mort du roi Arthur. Roman publié d'après le manuscrit de Lyon, Palais des Arts 77, complété par le manuscrit BnF n.a.fr. 1119. Édition bilingue. Publication, traduction, présentation et notes par Emmanuèle BAUMGARTNER et Marie-Thérèse DE MEDEIROS, Paris, Champion, 2007 («Champion Classiques Moyen Âge», 20), pp. 537.

1 La Mort le roi Artu est un roman grandiose, et l'on ne peut que se réjouir de le voir publié dans cette collection bilingue de la maison Champion. Il s'agit d'une nouvelle édition, fondée sur un manuscrit différent de celui qu'avait choisi Jean Frappier en 1936 (A: Arsenal 3347); dans la riche tradition du texte, qui compte 52 témoins y compris les fragments, c'est $K$ (Lyon, Palais des Arts 77) qui fournit maintenant le texte de base: s'agissant d'un manuscrit acéphale, les 58 premiers paragraphes ont été édités à partir de $Z$ (BnF n.a.f. 1119, comme on le lit sur la couverture et la page de titre, et non pas fr. 1119, comme il est indiqué à la p. 11 de l'«Introduction»), alors que, pour le texte contenu dans les deux derniers feuillets, endommagés, les éditrices ont eu recours au ms. 02 (Oxford, Bodleian Library Rawlison D.899) contrôlé sur les manuscrits appartenant à la même famille (c'est la famille $\mathrm{z}$ de Frappier: on se rapportera à la note 3, p. 458, puisque ces informations ne sont pas données aux pp. 12-13).

2 L'«Introduction» justifie d'abord la nouvelle édition, propose quelques remarques sur les manuscrits et explicite les raisons qui ont présidé au choix du manuscrit de Lyon. Dans le paragraphe consacré au traitement du texte, manifestement destiné aux non 
spécialistes, on ne peut pas manquer de relever une affirmation étonnante: si les manuscrits de la famille $\mathrm{z}$ «ne témoignent pas d'un strict respect de la déclinaison» ( $\mathrm{p}$. 14), les éditrices ont opté pour une normalisation rigoureuse, choix contestable à mes yeux puisqu'il fausse sous une prétendue régularité une variation déjà amplement à l'œuvre au XIII ${ }^{\text {e }}$ siècle. Une analyse détaillée du texte (pp. 18-28) est suivie de l'étude littéraire (pp. 28-36), qui privilégie le motif de la 'fin' (fin du cycle du Lancelot-Graal, mais aussi fin des aventures, fin des personnages) et les thèmes portants du roman: «mescheance et pechié», à savoir les causes de l'effondrement de l'univers arthurien, dans une certaine mesure compensés par "amour et amitié» (sans réhabiliter l'amour courtois, l'auteur se livre en effet à une magnifique célébration des liens d'amitié). Bien que nécessairement sélective, la Bibliographie occupe dix pages (pp. 37-46): on y trouvera tant un choix des éditions / traductions précédentes, que le concordancier dressé par Pierre Kunstmann et Martin Dubé (1982), et surtout les études sur la tradition manuscrite et sur l'œuvre (livres et articles séparés); l'indication «Norris, J. Lacy» (sic, p. 37) est évidemment fautive: le nom du savant américain apparaît heureusement sous sa forme exacte à la p. 43 («Lacy, Norris J.»); personnellement, je compléterais la bibliographie sur le motif du «don contraignant» par le renvoi à l'article de Jean Frappier (Le motif du 'don contraignant' dans la littérature du Moyen Âge, «Travaux de Linguistique et de Littérature», 7, 1969, pp. 7-46), article auquel réagit justement Philippe Ménard dans la contribution de 1981 citée ici p. 44 et dans les notes.

Le texte est bien édité, la traduction très agréable. L'apparat en bas de page est double: on trouvera, au-dessous du texte en ancien français, les remarques sur le manuscrit de base (leçons rejetées, corrections), au-dessous de la traduction les notes au texte (pour les observations linguistiques, il faudra alors rechercher les mots ou le passage commentés dans la version originale, ce qui n'est pas toujours aisé, en l'absence de renvois à la ligne du texte). Un choix des variantes relevées au sein de la famille $\mathrm{z}$ sont réunies aux pp. 473-494: cela ne rend pas facile la consultation, le lecteur intéressé étant contraint de passer constamment d'un apparat à l'autre.

4 On trouvera encore l'«Index» des noms propres aux pp. 495-500 et un «Glossaire» sélectif aux pp. 501-523; notons à ce propos qu'il s'agit d'un glossaire de seconde main, appuyé non pas sur les dictionnaires historiques, mais sur celui de Jean Frappier, et sur le glossaire de la Première Continuation de Perceval de Lucien Foulet (1955).

5 Le volume se clôt sur la transcription de la conférence qu'Emmanuèle Baumgartner avait présentée à Utrecht en juillet 2005, lors du XXI ${ }^{e}$ Congrès International de la Société Internationale Arthurienne, quelques jours seulement avant sa disparition («Lancelot et son clan», pp. 525-536): c'est un essai qui nous donne une dernière preuve - si besoin était - du savoir et de la sensibilité de Mme Baumgartner, et qui nous fait mesurer une fois de plus l'ampleur de sa perte. 